Check for updates

Cite this: Phys. Chem. Chem. Phys., 2020, 22, 12785

Received 24th November 2019, Accepted 22nd May 2020

DOI: 10.1039/c9cp06335h

rsc.li/pccp

\section{Vibrational mode frequency correction of liquid water in density functional theory molecular dynamics simulations with van der Waals correction $\dagger$}

\author{
Kai Zhong, ${ }^{\text {ab }}$ Chun-Chieh Yu, (D) ${ }^{\mathrm{b}}$ Mayank Dodia, ${ }^{\mathrm{b}}$ Mischa Bonn, (D) ${ }^{\mathrm{b}}$ \\ Yuki Nagata (D)*b and Tatsuhiko Ohto*c
}

\begin{abstract}
The frequencies and spectral lineshapes of the stretch and bending modes of water provide invaluable information on the microscopic structures of water in aqueous solutions and at the water/solid interfaces. Density functional theory molecular dynamics (DFT-MD) simulation has been used not only for predicting the properties of water but also for interpreting the vibrational spectra of water. Since the accuracy of the DFT-MD simulations relies on the choice of the exchange-correlation functionals and dispersion correction schemes employed, the predicted vibrational spectra at different levels of DFT theory differ significantly, prohibiting precise comparison of simulated spectra with experimental data. Here, we simulate the vibrational density of states for liquid heavy water based on various DFT-MD trajectories. We find that DFT-MD simulations tend to predict excessive inhomogeneous broadening for the stretch mode of water. Furthermore, we develop a frequency correction scheme for the stretch and bending modes of liquid water, which substantially improves the prediction of the vibrational spectra.
\end{abstract}

\section{Introduction}

The molecular structure of water is simple, yet the ensemble of water molecules leads to unique properties of liquid water, such as the higher density in the liquid phase than in the solid phase and large surface tension of water. These unique properties arise from the complex hydrogen-bond (H-bond) network of water. Understanding the relationship between the local conformation of water and $\mathrm{H}$-bond strength is essential for clarifying the properties of various aqueous systems.

Information on the $\mathrm{H}$-bond of water has been gained through vibrational spectroscopy such as infrared (IR) and Raman spectroscopy. ${ }^{1,2}$ Typically, the IR spectrum of liquid water consists of three major bands; the $\mathrm{O}-\mathrm{H}$ stretch mode, $\mathrm{H}-\mathrm{O}-\mathrm{H}$ bending mode, and librational modes of water. The $\mathrm{O}-\mathrm{H}$ stretch and $\mathrm{H}-\mathrm{O}-\mathrm{H}$ bending modes have been widely used as

\footnotetext{
${ }^{a}$ Hefei National Laboratory for Physical Sciences at the Microscale, Collaborative Innovation Center of Chemistry for Energy Materials, CAS Center for Excellence in Nanoscience, School of Chemistry and Materials Science, University of Science and Technology of China, Hefei, Anhui 230026, P. R. China

${ }^{b}$ Max Planck Institute for Polymer Research, Ackermannweg 10, 55128 Mainz, Germany.E-mail: nagata@mpip-mainz.mpg.de

${ }^{c}$ Graduate School of Engineering Science, Osaka University, 1-3 Machikaneyama, Toyonaka, Osaka 560-8531, Japan. E-mail: ohto@molectronics.jp

† Electronic supplementary information (ESI) available. See DOI: 10.1039/ c9cp06335h
}

reporters of the H-bond strength. ${ }^{3-6}$ When the $\mathrm{H}$-bond of water becomes stronger, the frequency of the $\mathrm{O}-\mathrm{H}$ stretch mode is lowered $^{4}$ and the frequency of the $\mathrm{H}-\mathrm{O}-\mathrm{H}$ bending mode is elevated. ${ }^{7}$ As such, frequency shifts of the $\mathrm{O}-\mathrm{H}$ stretch mode in aqueous solutions reflect how the solute-water interactions alter the strength and structure of the H-bond network of water. ${ }^{7-10}$ The target of the vibrational spectroscopy of water can be further expanded, when the vibrational technique is combined with a surface-specific technique. ${ }^{11-13}$ As such, vibrational spectroscopy becomes a valuable technique for probing the microscopic structure of interfacial water.

There are, however, several challenges associated with interpreting the vibrational spectra of water. First of all, the vibrational bands of the water spectra are often broad and featureless, making it difficult to assign specific features to the molecular conformations. Secondly, for more complex, composite aqueous solutions, it is often not clear which interaction is leading to which feature of the vibrational spectra. Simulating vibrational spectra based on the trajectories sampled by molecular dynamics (MD) simulation is a direct and powerful route to interpret the vibrational spectra, because it allows us to identify the contribution of water molecules in the bulk and near the solute molecules. ${ }^{5,14-18}$

When a solute-water interaction is complicated, developing accurate force field models for computing the vibrational spectra is not straightforward. In contrast with MD simulations 
using force field models, a density functional theory (DFT)-MD simulation computes the forces acting on the atoms based on DFT, which allows us to simulate complex solute-water interactions without using force field models. ${ }^{19-28}$ However, the predictive powers of DFT-MD simulations regarding the water properties rely heavily on the choice of the exchange-correlation (XC) functionals and van der Waals (vdW) corrections. ${ }^{29-35}$ Likewise, the peak frequencies in the vibrational spectra of liquid water are strongly affected by the XC functionals and vdW corrections. $^{36-38}$

To correct the peak frequency, one can use the dataset of scaling factors that are available for many XC functionals. ${ }^{39-41}$ These datasets, however, do not work well for water vibrational spectroscopy, as these datasets are often created to correct for gas-phase frequencies. Furthermore, the scaling factors are generally parametrized for a set of small molecules, which may provide inaccurate numerical values for the liquid water peak frequencies. As such, generating the correction scheme for the vibrational frequency of water is urgently required for achieving reliable interpretation of the vibrational spectra of water, based on the DFT-MD simulation with available XC functionals and vdW corrections. Note that, although the vibrational spectra will be affected by several factors including nuclear quantum effects, ${ }^{42,43}$ the scheme for correcting the frequency being presented here include these effects only implicitly; the correction factor provides a practical approach to estimate the frequency of liquid water, while accurate highlevel simulation requires nuclear quantum simulation, highlevel XC functional, and accurate basis sets, resulting in a computationally expensive simulation.

In this work, we calculated the vibrational density of states (VDOS) spectra for bulk heavy water $\left(\mathrm{D}_{2} \mathrm{O}\right)$ with various DFT methods and vdW methods. We find that the O-D stretch frequency is highly sensitive to the XC functionals and vdW correction schemes. In particular, DFT-MD simulations tend to predict a broader peak than the experimental data, indicating that DFT-MD describes an excessive inhomogeneous environment of bulk water. On the other hand, the D-O-D bending frequency is rather insensitive to the choice of DFT methods. Furthermore, we developed a frequency correction scheme to reproduce the stretch and bending peaks for water. These correction factors are employed to evaluate the heterogeneity of the O-D stretch mode of heavy water.

\section{Simulation procedures}

\subsection{Trajectories}

2.1.A. Born-Oppenheimer MD. We used the DFT-MD trajectories, which were generated in our previous papers. ${ }^{32-34}$ The BOMD simulations employed the QUICKSTEP method ${ }^{44}$ implemented in the CP2K program. ${ }^{45}$ We used the $\mathrm{PBE},{ }^{46} \mathrm{BLYP},{ }^{47,48}$ and revPBE ${ }^{49}$ GGA exchange-correlation (XC) functionals, M06-L ${ }^{50}$ and $\mathrm{B} 97 \mathrm{M}-\mathrm{rV}^{51}$ meta-GGA XC functionals, and B3LYP, ${ }^{47,52-54}$ revPBE0, ${ }^{49,55}$ and HSE06 ${ }^{56,57}$ hybrid-GGA XC functionals for systematic investigation of the DFT methods. For the hybrid-GGA
DFT-MD simulations, we utilized the auxiliary density matrix method (ADMM), ${ }^{58}$ which reduces the computational cost for hybrid-GGA calculations. To include the dispersion energy missing in DFT scheme, we utilized Grimme's empirical D2, ${ }^{59}$ $\mathrm{D} 3(0),{ }^{60} \mathrm{D} 3(\mathrm{BJ}),{ }^{61}$ and $\mathrm{D} 3 \mathrm{~m}(\mathrm{BJ})^{62}$ vdW corrections, as well as the non-local (DRSLL ${ }^{63,64}$ and rVV10 ${ }^{65,66}$ ) vdW corrections. The revPBE0-D3(0) simulations were performed with and without the ADMM.

We used the mixed Gaussian and plane wave approach as implemented in the $\mathrm{CP} 2 \mathrm{~K}$ code. For the Gaussian part, the TZV2P basis set which is constructed using triple-zeta valence Gaussian basis with two sets of polarization functions was used for all BOMD simulations. We set the plane wave density cutoff of $320 \mathrm{Ry}$. Only for the M06-L-D3(0), we used a cutoff of $1200 \mathrm{Ry}$, because the M06-L functional requires a finer integration grid. ${ }^{43}$ Norm-conserving Goedecker-Teter-Hutter pseudopotentials ${ }^{67,68}$ were used to describe the core electrons. To accelerate the MD simulations, $\mathrm{D}_{2} \mathrm{O}$ was used instead of $\mathrm{H}_{2} \mathrm{O}$, and the time step was set to 0.5 fs. We simulated $160 \mathrm{D}_{2} \mathrm{O}$ molecules in the $16.63 \AA \times 16.63 \AA \times 44.10 \AA$ cell in a slab configuration at $300 \mathrm{~K}$ in the $N V T$ ensemble. The canonical sampling through velocity rescaling $\operatorname{method}^{69}$ was employed as the thermostat. The cutoff radius of the vdW interactions was set to $10 \AA$.

We ran 10 independent simulations from previously generated configurations from the simulation at the revPBE-D3(0) level of theory. ${ }^{32}$ From each simulation, a 16 ps MD trajectory was generated after 5 ps equilibration run. The length of the trajectories were total $\geq 500$ ps for BLYP, BLYP-D2, BLYP-D3(0), BLYP-D3(BJ), PBE, PBE-D2, PBE-D3(0), PBE-D3(BJ), revPBED3(0) and revPBE-D3(BJ) GGA functionals, $\geq 150$ ps for PBED3m(BJ), PBE-DRSLL, PBE-rVV10, optB88-DRSLL, revPBE-D2, revPBE-DRSLL, revPBE-rVV10 GGA functionals, M06-L-D3(0), B97M-rV meta-GGA functionals, B3LYP-D3(0), HSE06-D3(0), revPBE0-D3(0) hybrid-GGA functionals, and $160 \mathrm{ps}$ for revPBE-D3(0) hybrid functionals without ADMM.

2.1.B. Car-Parrinello MD (CPMD). For the SCAN meta-GGA $\mathrm{XC}$ functional, ${ }^{70,71}$ we employed the CPMD methodology using the Quantum Espresso code. ${ }^{72,73}$ We simulated $128 \mathrm{D}_{2} \mathrm{O}$ molecules in a $12.44 \AA \times 12.44 \AA \times 50 \AA$ cell. We employed the Hamann-Schlüter-Chiang-Vanderbilt pseudopotentials ${ }^{74,75}$ generated for PBE with a plane-wave cutoff of 85 Ry and a time step of 2 a.u. (0.0484 fs). The simulation was performed at $300 \mathrm{~K}$ in NVT ensemble with the Nosé-Hoover chain thermostat. $^{76,77}$ The fictitious mass of electrons was set at 100 a.u. $^{78,79}$ The 56 ps CPMD trajectory was generated after 5 ps equilibration run.

2.1.C. Classical MD. The POLI2VS model of water is known to reproduce the IR and Raman spectra of bulk liquid water as well as the SFG spectra at the water-air interface (see ESI $\dagger$ ). ${ }^{80-82}$ Based on this excellent performance of the POLI2VS model, we used the POLI2VS model of water as the reference and calculated the VDOS spectra based on the POLI2VS MD trajectories. We performed the classical MD simulation with POLI2VS model for $160 \mathrm{D}_{2} \mathrm{O}$ molecules in the $16.63 \AA \times 16.63 \AA \times 44.10 \AA$ cell in a slab configuration at $300 \mathrm{~K}$ by using the Nosé-Hoover chain 

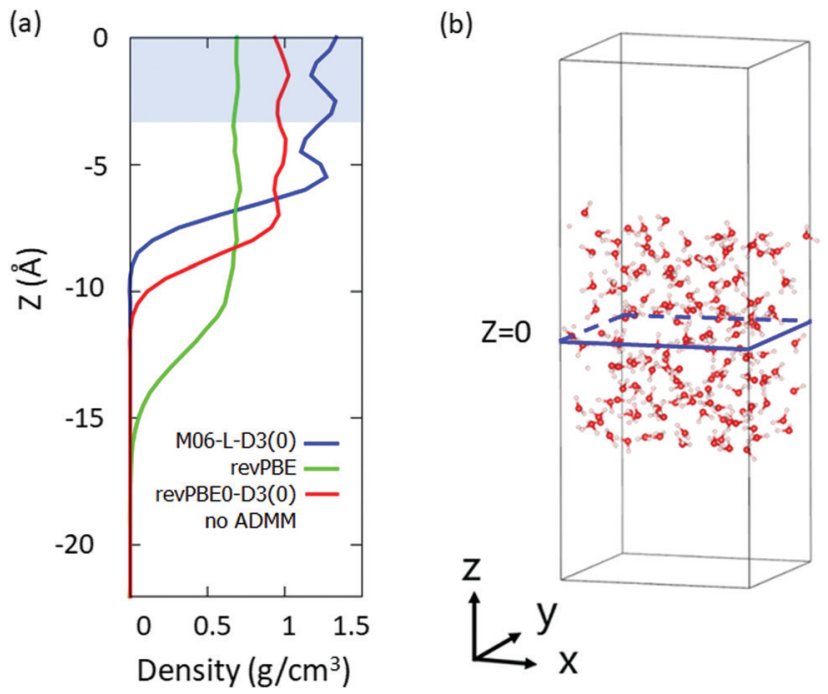

Fig. 1 (a) Density profiles of $\mathrm{D}_{2} \mathrm{O}$ molecules simulated at the M06-L$D 3(0)$, revPBE and revPBEO-D3(0) (with no ADMM) levels of theory. The highlighted region indicates the volume used for the calculations. (b) Snapshot of the DFT-MD trajectory at the revPBE-D3(0) level of theory.

thermostat ${ }^{76,77}$ in $N V T$ ensemble. The time step for integrating the equations of motion was 0.4 fs. The charge-charge, charge-dipole and dipole-dipole interactions were evaluated using Ewald summation, whereas quadrupole interactions were curtailed at $8.3 \AA$ A. We first ran 700 ps MD simulation for equilibration and then we obtained 1 ns for production run which was used for analysis.

\subsection{VDOS spectra calculation based on MD trajectories}

VDOS is computed for the bulk $\mathrm{D}_{2} \mathrm{O}$ molecules by using the velocity-velocity autocorrelation:

$$
\operatorname{VDOS}(\omega)=\int_{0}^{T} \mathrm{~d} t \cos (\omega t) \cos ^{2}\left(\frac{\pi t}{2 T}\right) \sum_{i} m_{i}\left(\boldsymbol{v}_{i}(0) \cdot \boldsymbol{v}_{i}(t)\right)
$$

where $\boldsymbol{v}_{i}(t)$ denotes the velocity vector of the atom $i$ at time $t, m_{i}$ is the mass of the atom $i, T$ is the length of the time correlation function. We set $T$ to $1 \mathrm{ps}$. The computed spectrum with the POLI2VS model shows response at frequencies higher than those observed in the experimental data. The higher frequencies of the POLI2VS model arise from the lack of nuclear quantum effects. ${ }^{82}$ Thus, we scaled the frequency axis with a factor of 0.96 for both the O-D stretch and the D-O-D bending modes (see ESI $\dagger$ ). ${ }^{83}$

We selected the bulk $\mathrm{D}_{2} \mathrm{O}$ molecules as a $\mathrm{D}_{2} \mathrm{O}$ molecule with the $z$-coordinate of its oxygen atom $|z|<3 \AA$, where we set the origin point of $z=0$ as a center of mass of the system. The region which we used for the VDOS calculation is highlighted in Fig. 1(a), while the snapshot of the simulation is drawn in Fig. 1(b).

\section{Results}

\subsection{VDOS Spectra}

Fig. 2 displays the calculated VDOS spectra with various combinations of the XC functionals and the $\mathrm{vdW}$ corrections, together with the reference POLI2VS data. Fig. 2 demonstrates
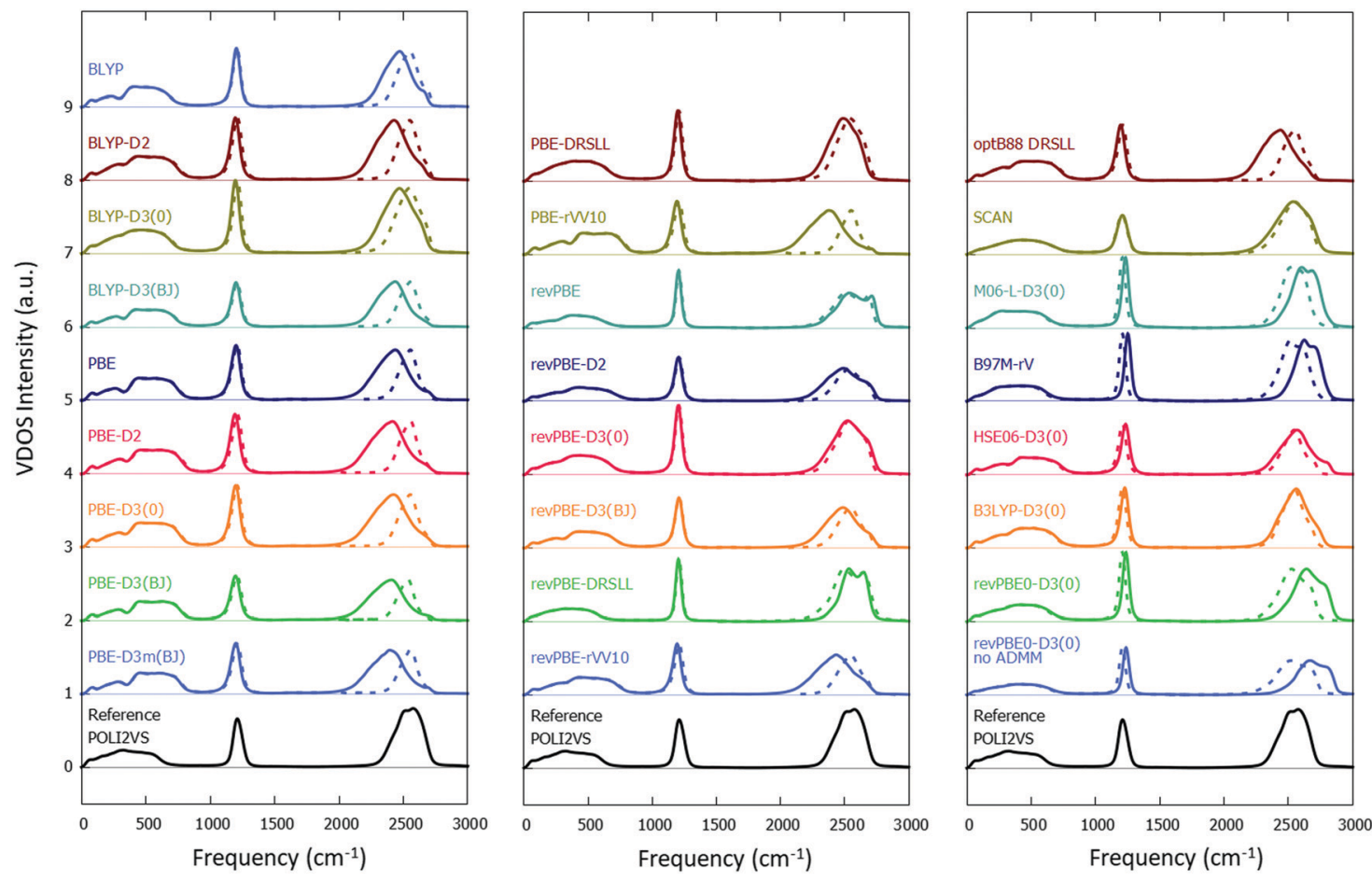

Fig. 2 Simulated VDOS spectra for $\mathrm{D}_{2} \mathrm{O}$ with different DFT methods. The solid and dotted lines represent the simulated VDOS spectra without frequency correction and with frequency correction via eqn (2) and (4), respectively. The reference data was obtained from the POLI2VS model. 
that the spectral shapes of the O-D stretch mode are very sensitive to the choice of the DFT functional, whereas the D-O-D bending mode is comparatively insensitive. When we focus on the linewidth of the $\mathrm{O}-\mathrm{D}$ stretch peak, one can recognize that the VDOS spectra computed from the DFT-MD trajectories are generally broader than the reference VDOS spectra. For example, the VDOS spectra computed from the revPBE0-D3(0) and SCAN functionals show much broader FWHMs (299 and $327 \mathrm{~cm}^{-1}$, respectively) than the reference POLI2VS data (FWHM of $259 \mathrm{~cm}^{-1}$ ). Since the linewidths of the spectra arise mainly from inhomogeneous broadening, this excessive broadening of the $\mathrm{O}-\mathrm{D}$ stretch peak indicates that the DFT-MD simulations tend to sample a larger variety of the $\mathrm{H}$-bond strengths than classical MD simulation.

The comparison between the FWHM of the VDOS obtained from DFT-MD simulations and classical MD simulation is sharply contrasted with the comparison of their respective simulated IR spectra. Specifically, the reported linewidths of the IR stretch mode spectra using the revPBE0-D3(0) and SCAN functionals (FWHMs of $251 \mathrm{~cm}^{-136}$ and $246 \mathrm{~cm}^{-1,37}$ respectively) exhibit good agreement with the simulated IR spectra (FWHM of $257 \mathrm{~cm}^{-182}$ ). These different trends in the VDOS spectra and IR spectra can be explained as follows. The linewidth of the IR spectrum of neat $\mathrm{D}_{2} \mathrm{O}$ is determined not only by the inhomogeneous broadening but also by the intermolecular/ intramolecular vibrational coupling ${ }^{1,5,84}$ as well as the frequencydependent transition dipole moment. ${ }^{85}$ Thus, the good agreement between the FWHMs in the IR spectra and poor agreement between the FWHMs in the VDOS data indicates that the FWHM of the IR spectra is dominated by the inhomogeneous broadening in the DFT-MD simulations, while in the classical MD simulations, the FWHM is largely determined by intermolecular/intramolecular vibrational coupling as well as the frequency-dependent transition dipole moment.

Among the DFT-MD simulations considered in this study, the B97M-rV and M06-L-D3(0) levels of theory provide reasonable linewidths of the O-D stretch mode, in line with ref. 42 . However, we also reported that these methods are inadequate for describing the water properties and the surface-specific vibrational spectra of water. ${ }^{34}$ This means that there is room for improving the XC functionals and vdW corrections. Finally, we note that, although a small $1500 \mathrm{~cm}^{-1}$ peak can be seen in the experimental data, it cannot be properly captured in the VDOS data, as this peak arises from the combination of bending and librational motions, and the VDOS calculation cannot capture such combination band properly. A high-level computational scheme would be needed to reproduce this combination band of water. ${ }^{86,87}$

\subsection{Frequency correction factor}

To correct the bandwidth as well as the peak position, we developed a correction scheme for the O-D stretch mode of $\mathrm{D}_{2} \mathrm{O}$. For correcting the frequency, we assumed the expression as;

$$
\omega_{\text {str,ref }}=a \omega_{\text {str,DFT-MD }}+b
$$

Table 1 The simulated characteristic frequencies for the O-D stretch mode and peak frequencies for the D-O-D bending mode in the VDOS spectra as well as the free O-D stretch frequency in the SFG spectra ${ }^{34,89}$

\begin{tabular}{llll}
\hline DFT methods & $\begin{array}{l}\omega_{\text {str }} \\
\left(\mathrm{cm}^{-1}\right)\end{array}$ & $\begin{array}{l}\text { Free O-D } \\
\text { frequency }\left(\mathrm{cm}^{-1}\right)\end{array}$ & $\begin{array}{l}\omega_{\text {bend }} \\
\left(\mathrm{cm}^{-1}\right)\end{array}$ \\
\hline BLYP & 2458 & 2688 & 1204 \\
BLYP-D2 & 2413 & 2688 & 1194 \\
BLYP-D3(0) & 2465 & 2688 & 1194 \\
BLYP-D3(BJ) & 2410 & 2687 & 1201 \\
PBE & 2411 & 2725 & 1201 \\
PBE-D2 & 2381 & 2725 & 1191 \\
PBE-D3(0) & 2399 & 2723 & 1197 \\
PBE-D3(BJ) & 2369 & 2718 & 1197 \\
PBE-D3m(BJ) & 2375 & 2718 & 1197 \\
PBE-DRSLL & 2490 & 2687 & 1199 \\
PBE-rVV10 & 2357 & 2712 & 1194 \\
revPBE & 2569 & 2740 & 1204 \\
revPBE-D2 & 2495 & 2732 & 1204 \\
revPBE-D3(0) & 2530 & 2737 & 1204 \\
revPBE-D3(BJ) & 2481 & 2732 & 1207 \\
revPBE-DRSLL & 2567 & 2697 & 1204 \\
revPBE-rVV10 & 2424 & 2712 & 1194 \\
optB88-DRSLL & 2417 & 2692 & 1194 \\
SCAN & 2527 & 2738 & 1213 \\
M06-L-D3(0) & 2628 & 2699 & 1234 \\
B97M-rV & 2645 & 2805 & 1251 \\
HSE06-D3(0) & 2562 & 2819 & 1234 \\
B3LYP-D3(0) & 2554 & 2773 & 1227 \\
revPBE0-D3(0) & 2657 & 2837 & 1237 \\
revPBE0-D3(0) no ADMM & 2686 & 2847 & 1237 \\
Reference & 2540 & 2714 & 1209 \\
& & &
\end{tabular}

where $\omega_{\text {str,ref }}$ is the characteristic frequency of the reference VDOS spectrum obtained from POLI2VS model and $\omega_{\text {str,DFT-MD }}$ is the VDOS spectrum frequency obtained from the DFT-MD trajectory. We used two parameters, $a$ and $b$, because the O-D stretch frequency varies largely and thus a single scale factor is insufficient to capture the trend of the stretch mode of $\mathrm{D}_{2} \mathrm{O} .^{88}$ To obtain the characteristic frequency, we computed the first moment of the VDOS;

$$
\omega_{\text {str,DFT-MD }}=\frac{\int_{\omega_{1}}^{\omega_{2}} \omega \operatorname{VDOS}_{\text {DFT-MD }}(\omega) \mathrm{d} \omega}{\int_{\omega_{1}}^{\omega_{2}} \operatorname{VDOS}_{\mathrm{DFT}-\mathrm{MD}}(\omega) \mathrm{d} \omega}
$$

where $\omega_{1}$ and $\omega_{2}$ are the frequencies at which the VDOS intensities are $10 \%$ of the maximum intensity of the $\mathrm{O}-\mathrm{D}$ stretch peak. The data is displayed in Table 1.

To determine two parameters, $a$ and $b$, one needs to have the additional relation of $\omega_{\text {str,ref }}$ with $\omega_{\text {str,DFT-MD }}$. This relation can be obtained from the peak frequency of the free O-D stretch in the DFT-MD simulated and experimental sum-frequency generation (SFG) spectra. At the free surface of water, a small fraction of water molecules finds itself with one non-hydrogen bonded $\mathrm{OH} / \mathrm{OD}$ group that sticks into the vapor phase. The frequency of this free $\mathrm{OH} / \mathrm{OD}$ group can be determined using sum-frequency generation spectroscopy. The free O-D stretch frequency of $2714 \mathrm{~cm}^{-1}$ is obtained from ref. 89, while the simulated free $\mathrm{O}-\mathrm{D}$ stretch frequencies using DFT-MD simulations were obtained from ref. 34 . The results are summarized in Table 1. 
Table 2 The correction factors for the O-D stretch mode and D-O-D bending mode

\begin{tabular}{llrl}
\hline DFT methods & $a$ & $b\left(\mathrm{~cm}^{-1}\right)$ & $c$ \\
\hline BLYP & 0.757 & 679 & 1.004 \\
BLYP-D2 & 0.633 & 1013 & 1.013 \\
BLYP-D3(0) & 0.780 & 617 & 1.013 \\
BLYP-D3(BJ) & 0.628 & 1027 & 1.007 \\
PBE & 0.554 & 1204 & 1.007 \\
PBE-D2 & 0.506 & 1335 & 1.015 \\
PBE-D3(0) & 0.537 & 1252 & 1.010 \\
PBE-D3(BJ) & 0.499 & 1358 & 1.010 \\
PBE-D3m(BJ) & 0.507 & 1336 & 1.010 \\
PBE-DRSLL & 0.883 & 341 & 1.008 \\
PBE-rVV10 & 0.490 & 1385 & 1.013 \\
revPBE & 1.018 & -75 & 1.004 \\
revPBE-D2 & 0.734 & 709 & 1.004 \\
revPBE-D3(0) & 0.841 & 412 & 1.004 \\
revPBE-D3(BJ) & 0.693 & 821 & 1.002 \\
revPBE-DRSLL & 1.338 & -895 & 1.004 \\
revPBE-rVV10 & 0.604 & 1076 & 1.013 \\
optB88-DRSLL & 0.633 & 1010 & 1.013 \\
SCAN & 0.825 & 455 & 0.997 \\
M06-L-D3(0) & 0.946 & 54 & 0.980 \\
B97M-rV & 1.088 & -338 & 0.966 \\
HSE06-D3(0) & 0.677 & 806 & 0.980 \\
B3LYP-D3(0) & 0.791 & 520 & 0.985 \\
revPBE0-D3(0) & 0.967 & -29 & 0.977 \\
revPBE0-D3(0) no ADMM & 1.081 & -364 & 0.977 \\
& & &
\end{tabular}

From the relation for the VDOS O-D stretch data and the free O-D stretch data, we obtained the parameters $a$ and $b$. The parameters are listed in Table 2, while the O-D stretch spectra modified by using eqn (2) are plotted with the broken lines in Fig. 2. The frequency-corrected VDOS spectra of the O-D stretch mode show close agreement with the reference data. This illustrates that the frequency correction scheme is powerful for predicting the vibrational frequency and lineshape of the O-D stretch mode.

We further obtained the correction factor $c$ for the D-O-D bending mode via;

$$
\omega_{\text {bend,ref }}=c \omega_{\text {bend,ref,DFT-MD }}
$$

where the bending mode peak frequency in the VDOS spectra with the DFT-MD simulation is $\omega_{\text {bend,ref,DFT-MD }}$ and the reference POLI2VS VDOS spectrum is $\omega_{\text {bend,ref. The obtained para- }}$ meters are also given in Table 1 . In contrast with the $\mathrm{O}-\mathrm{D}$ stretch mode, the peak frequency and the lineshape of the simulated $\mathrm{D}-\mathrm{O}-\mathrm{D}$ bending mode spectra are insensitive to the choice of specific DFT method and conform with the reference spectra.

These correction factors can be also used to predict the vibrational spectra of $\mathrm{H}_{2} \mathrm{O}$ by scaling the vibrational frequency axis with the factor of $1 / 0.735$ in the $\mathrm{D}_{2} \mathrm{O}$ spectra. ${ }^{89}$ In fact, the vibrational spectra lineshapes of $\mathrm{H}_{2} \mathrm{O}$ and $\mathrm{D}_{2} \mathrm{O}$ become quite similar after the frequency axis is scaled by this factor. ${ }^{90}$ Finally, we note that our correction scheme include a red-shift induced by nuclear quantum effects. For the DFT-MD simulation with the nuclear quantum effects such as the ring-polymer MD simulation $^{91}$ and centroid MD simulation, ${ }^{92}$ we recommend using the coefficients, $a$ and $c$, divided by a factor of 0.96 .

\subsection{Decomposition of the $\mathrm{O}-\mathrm{D}$ stretch spectra}

By establishing the frequency correction scheme for the DFT-MD trajectories, we can compare the individual spectra simulated at different DFT levels. Here, we investigate the level of heterogeneity of the O-D stretch spectra at different DFT levels of theory. To do so, we decomposed the spectra based on
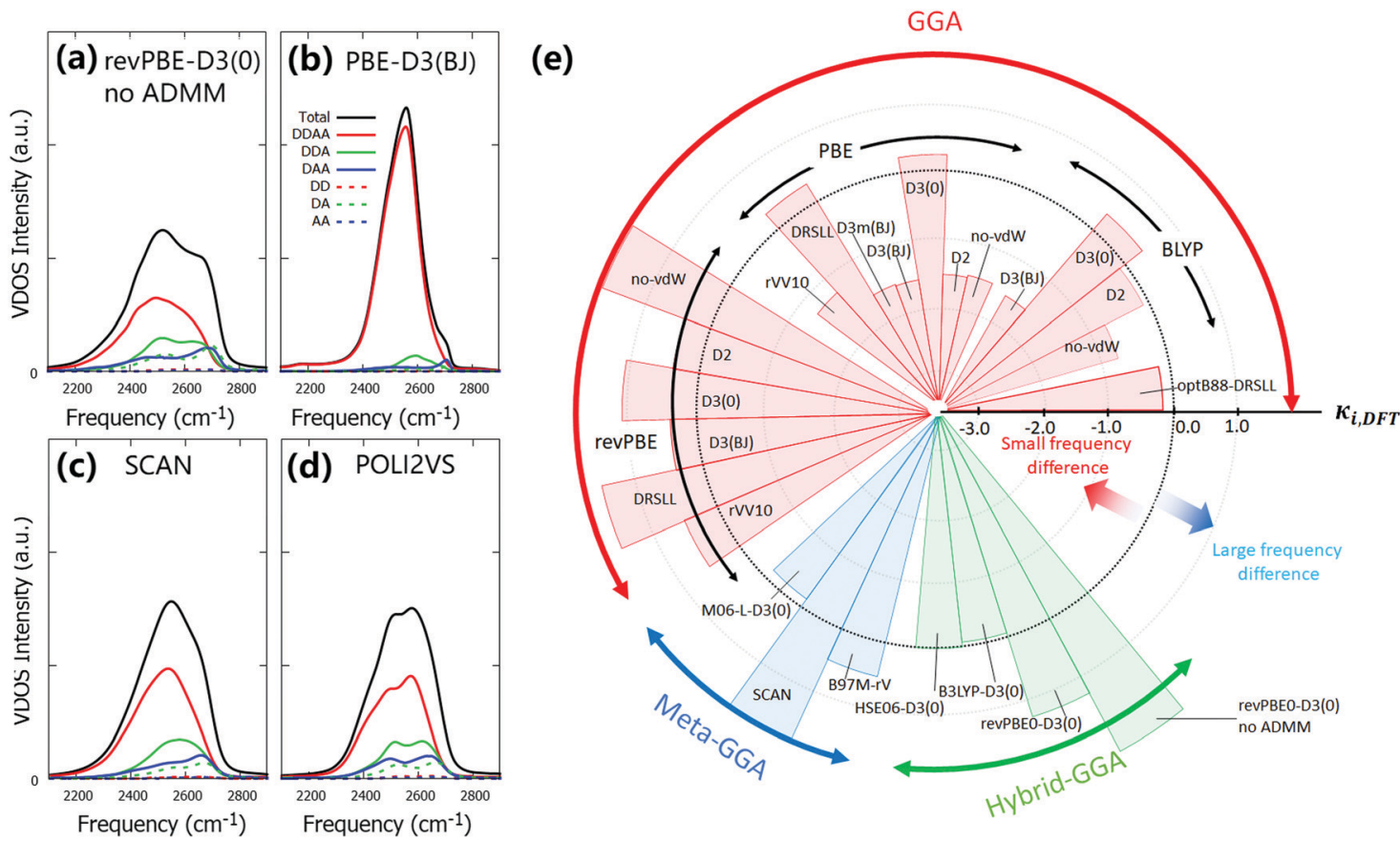

Fig. 3 Decomposed VDOS spectra (a) at the revPBEO-D3(0) without ADMM level of theory, (b) at the PBE-D3(BJ) level of theory, and (c) at the SCAN level of theory as well as (d) with the POLI2VS model. (e) Pie chart of averaged $\kappa_{i, \text { DFT }}$ for various DFT methods. 
the number of donating and accepting hydrogen bonds (DDAA, DDA, DAA, DD, DA, AA, and others). The hydrogen bond formation was judged at time $t=0$ in eqn (1) via the geometry of water dimer; when the $\mathrm{O} \cdots \mathrm{O}$ distance is less than $3.5 \AA$ and the $\mathrm{O} \cdots \mathrm{O}-\mathrm{D}$ angle is less than 30 degrees, the hydrogen bond is formed. ${ }^{93}$

A few representative frequency-corrected DFT-MD spectra with O-D stretch decomposition are shown in Fig. 3(a)-(d). The computed spectra with other DFT methods are given in the ESI. $\dagger$ The data show that the O-D stretch mode is dominated by the contribution of the DDAA species, while the other water species, such as DDA and DAA, also contribute to the water spectra. The DDAA species constitute the lowest frequency component, and the water species with a smaller number of the hydrogen bond acceptor or donor provide higher frequency component, as is expected. ${ }^{4}$ However, one can see a marked difference of the frequency shift between the DDAA and the DDA/DAA/DD/DA/AA species. To quantify this, we computed the quantities of

$$
\begin{gathered}
\Delta \omega_{i, \mathrm{DFT}}=\omega_{i, \mathrm{DFT}}-\omega_{\mathrm{DDAA}} \\
\kappa_{i, \mathrm{DFT}}=\left(\Delta \omega_{i, \mathrm{DFT}}-\Delta \omega_{i, \mathrm{ff}}\right) / \Delta \omega_{i, \mathrm{sd}}
\end{gathered}
$$

where $\omega_{i, \mathrm{DFT}}$ is the center of mass frequency via eqn (3) for $i=$ DDA, DAA, DD, DA, AA. We set $\Delta \omega_{i \text {,ff }}$ and $\Delta \omega_{i, \text { sd }}$ as the frequency differences, and the standard deviation for the POLI2VS data computed from DFT-MD data, respectively. The average of $\kappa_{i, \text { DFT }}$ is shown in the pie chart of Fig. 3(e). This clearly indicates that one can see the marked variation of the heterogeneity of O-D stretch spectra. The BLYP and PBE levels of theory tend to predict homogeneous water than the classical MD simulations. Among these, the description highly depends on the functionals. The HSE06-D3(0), B3LYP-D3(0), revPBE-D2, revPBE-D3(BJ) and BLYP-D2 methods show small deviations from the reference POLI2VS data.

\section{Conclusion}

We have computed the VDOS spectra of heavy water for the DFT-MD trajectories with various DFT methods and compared them with the reference data. We found that different DFT methods provide a variety of the O-D stretch mode peak frequency and linewidth in the VDOS spectra. In particular, the VDOS spectra of the DFT-MD simulations tend to show a broader linewidth than the reference POLI2VS VDOS spectrum, indicating that the DFT-MD simulations excessively sample the inhomogeneous environments of water stretch mode. Subsequently, we developed the frequency correction scheme for the stretch mode and bending mode. Our data shows that the correction proposed here can improve the prediction and interpretation of the vibrational spectra of $\mathrm{D}_{2} \mathrm{O}$. By further scaling the frequency axis of the O-D stretch mode to the $\mathrm{O}-\mathrm{H}$ stretch mode via a factor of $1 / 0.735$, one can also predict the $\mathrm{O}-\mathrm{H}$ stretch spectra reasonably.

The correction scheme was further applied to evaluate the homogeneity of the O-D stretch spectra. By decomposing the corrected VDOS into the contributions from differently hydrogen-bonded water species, we revealed that the HSE06D3(0), B3LYP-D3(0), revPBE-D2, revPBE-D3(BJ) and BLYP-D2 levels of theory predict a reasonable homogeneity of water frequencies with the classical MD simulation.

\section{Conflicts of interest}

There are no conflicts to declare.

\section{Acknowledgements}

We acknowledge the financial support from MaxWater project of Max Planck Society. This research used computational resources of Oakforest-PACS provided by Multidisciplinary Cooperative Research Program in Center for Computational Sciences, University of Tsukuba. Open Access funding provided by the Max Planck Society.

\section{References}

1 F. Perakis, L. De Marco, A. Shalit, F. Tang, Z. R. Kann, T. D. Kühne, R. Torre, M. Bonn and Y. Nagata, Vibrational Spectroscopy and Dynamics of Water, Chem. Rev., 2016, 116, 7590-7607.

2 H. J. Bakker and J. L. Skinner, Vibrational Spectroscopy as a Probe of Structure and Dynamics in Liquid Water, Chem. Rev., 2010, 110, 1498-1517.

3 M. Falk, The Frequency of the H-O-H Bending Fundamental in Solids and Liquids, Spectrochim. Acta, Part A, 1984, 40, 43-48.

4 R. Rey, K. B. Møller and J. T. Hynes, Hydrogen Bond Dynamics in Water and Ultrafast Infrared Spectroscopy, J. Phys. Chem. A, 2002, 106, 11993-11996.

5 B. M. Auer and J. L. Skinner, IR and Raman Spectra of Liquid Water: Theory and Interpretation, J. Chem. Phys., 2008, 128, 224511.

6 Y. Ni and J. L. Skinner, IR and SFG Vibrational Spectroscopy of the Water Bend in the Bulk Liquid and at the LiquidVapor Interface, Respectively, J. Chem. Phys., 2015, 143, 014502.

7 L. Piatkowski and H. J. Bakker, Vibrational Dynamics of the Bending Mode of Water Interacting with Ions, J. Chem. Phys., 2011, 135, 214509.

8 J. Hunger, K. J. Tielrooij, R. Buchner, M. Bonn and H. J. Bakker, Complex Formation in Aqueous Trimethylamine-NOxide (TMAO) Solutions, J. Phys. Chem. B, 2012, 116, 4783-4795.

9 J. G. Davis, K. P. Gierszal, P. Wang and D. Ben-Amotz, Water Structural Transformation at Molecular Hydrophobic Interfaces, Nature, 2012, 491, 582-585.

10 R. Biswas, W. Carpenter, J. A. Fournier, G. A. Voth and A. Tokmakoff, IR Spectral Assignments for the Hydrated Excess Proton in Liquid Water, J. Chem. Phys., 2017, 146, 154507.

11 V. Ostroverkhov, G. A. Waychunas and Y. R. Shen, New Information on Water Interfacial Structure Revealed by 
Phase-Sensitive Surface Spectroscopy, Phys. Rev. Lett., 2005, 94, 046102.

12 A. Eftekhari-Bafrooei and E. Borguet, Effect of Surface Charge on the Vibrational Dynamics of Interfacial Water, J. Am. Chem. Soc., 2009, 131, 12034-12035.

13 H. C. Allen, N. N. Casillas-Ituarte, M. R. Sierra-Hernández, X. Chen and C. Y. Tang, Shedding Light on Water Structure at Air-Aqueous Interfaces: Ions, Lipids, and Hydration, Phys. Chem. Chem. Phys., 2009, 11, 5538-5549.

14 T. Yagasaki and S. Saito, Ultrafast Intermolecular Dynamics of Liquid Water: A Theoretical Study on Two-Dimensional Infrared Spectroscopy, J. Chem. Phys., 2008, 128, 154521.

15 S. Imoto, S. S. Xantheas and S. Saito, Molecular Origin of the Difference in the $\mathrm{HOH}$ Bend of the IR Spectra between Liquid Water and Ice, J. Chem. Phys., 2013, 138, 054506.

16 M. M. Skinner, A. A. Martin and B. C. Moore, Is Lipid Correction Necessary in the Stable Isotope Analysis of Fish Tissues?, Rapid Commun. Mass Spectrom., 2016, 30, 881-889.

17 K. Usui, J. Hunger, M. Sulpizi, T. Ohto, M. Bonn and Y. Nagata, Ab Initio Liquid Water Dynamics in Aqueous TMAO Solution, J. Phys. Chem. B, 2015, 119, 10597-10606.

18 T. Ohto, E. H. G. Backus, C.-S. Hsieh, M. Sulpizi, M. Bonn and Y. Nagata, Lipid Carbonyl Groups Terminate the Hydrogen Bond Network of Membrane-Bound Water, J. Phys. Chem. Lett., 2015, 6, 4499-4503.

19 Y. Nagata, S. Yoshimune, C. S. Hsieh, J. Hunger and M. Bonn, Ultrafast Vibrational Dynamics of Water Disentangled by Reverse Nonequilibrium Ab Initio Molecular Dynamics Simulations, Phys. Rev. X, 2015, 5, 021002.

20 C. Zhang, D. Donadio, F. Gygi and G. Galli, First Principles Simulations of the Infrared Spectrum of Liquid Water Using Hybrid Density Functionals, J. Chem. Theory Comput., 2011, 7, 1443-1449.

21 T. Ohto, K. Usui, T. Hasegawa, M. Bonn and Y. Nagata, Toward Ab Initio Molecular Dynamics Modeling for SumFrequency Generation Spectra; an e Cient Algorithm Based on Surface-Specific Velocity-Velocity Correlation Function, J. Chem. Phys., 2015, 143, 124702.

22 R. Iftimie and M. E. Tuckerman, The Molecular Origin of the "Continuous" Infrared Absorption in Aqueous Solutions of Acids: A Computational Approach, Angew. Chem., Int. Ed., 2006, 45, 1144-1147.

23 W. Chen, M. Sharma, R. Resta, G. Galli and R. Car, Role of Dipolar Correlations in the Infrared Spectra of Water and Ice, Phys. Rev. B: Condens. Matter Mater. Phys., 2008, 77, 245114.

24 H. S. Lee and M. E. Tuckerman, Dynamical Properties of Liquid Water from Ab Initio Molecular Dynamics Performed in the Complete Basis Set Limit, J. Chem. Phys., 2007, 126, 164501.

25 M. Sharma, R. Resta and R. Car, Intermolecular Dynamical Charge Fluctuations in Water: A Signature of the H-Bond Network, Phys. Rev. Lett., 2005, 95, 187401.

26 B. Das, B. Sharma and A. Chandra, Effects of tert-Butyl Alcohol on Water at the Liquid-Vapor Interface: Structurally Bulk-like but Dynamically Slow Interfacial Water, J. Phys. Chem. C, 2018, 122, 9374-9388.
27 G. Melani, Y. Nagata, J. Wirth and P. Saalfrank, Vibrational Spectroscopy of Hydroxylated $\alpha-\mathrm{Al}_{2} \mathrm{O}_{3}(0001)$ Surfaces with and without Water: An Ab Initio Molecular Dynamics Study, J. Chem. Phys., 2018, 149, 014707.

28 L. W. Pinkley, P. P. Sethna and D. Williams, Optical Constants of Water in the Infrared: Influence of Temperature, J. Opt. Soc. Am., 1977, 67, 494-499.

29 I.-C. Lin, A. P. Seitsonen, I. Tavernelli and U. Rothlisberger, Structure and Dynamics of Liquid Water from Ab Initio Molecular Dynamics-Comparison of BLYP, PBE, and RevPBE Density Functionals with and without van der Waals Corrections, J. Chem. Theory Comput., 2012, 8, 3902-3910.

30 M. J. Gillan, D. Alfè and A. Michaelides, Perspective: How Good Is DFT for Water?, J. Chem. Phys., 2016, 144, 130901.

31 S. Grimme, A. Hansen, J. G. Brandenburg and C. Bannwarth, Dispersion-Corrected Mean-Field Electronic Structure Methods, Chem. Rev., 2016, 116, 5105-5154.

32 T. Ohto, M. Dodia, S. Imoto and Y. Nagata, Structure and Dynamics of Water at the Water-Air Interface Using First-Principles Molecular Dynamics Simulations within Generalized Gradient Approximation, J. Chem. Theory Comput., 2019, 15, 595-602.

33 M. Dodia, T. Ohto, S. Imoto and Y. Nagata, Structure and Dynamics of Water at the Water-Air Interface Using FirstPrinciples Molecular Dynamics Simulations. II. NonLocal vs Empirical van der Waals Corrections, J. Chem. Theory Comput., 2019, 15, 3836-3843.

34 T. Ohto, M. Dodia, J. Xu, S. Imoto, F. Tang, F. Zysk, T. D. Kühne, Y. Shigeta, M. Bonn and X. Wu, et al., Accessing the Accuracy of Density Functional Theory through Structure and Dynamics of the Water-Air Interface, J. Phys. Chem. Lett., 2019, 10, 4914-4919.

35 J. G. Brandenburg, A. Zen, D. Alfè and A. Michaelides, Interaction between Water and Carbon Nanostructures: How Good Are Current Density Functional Approximations?, J. Chem. Phys., 2019, 151, 164702.

36 O. Marsalek and T. E. Markland, Quantum Dynamics and Spectroscopy of Ab Initio Liquid Water: The Interplay of Nuclear and Electronic Quantum Effects, J. Phys. Chem. Lett., 2017, 8, 1545-1551.

37 J. Xu, M. Chen, C. Zhang and X. Wu, First-Principles Study of the Infrared Spectrum in Liquid Water from a Systematically Improved Description of H-Bond Network, Phys. Rev. $B, 2019$, 99, 205123.

38 M. J. DelloStritto, S. M. Piontek, M. L. Klein and E. Borguet, Effect of Functional and Electron Correlation on the Structure and Spectroscopy of the $\mathrm{Al}_{2} \mathrm{O}_{3}$ (001)- $\mathrm{H}_{2} \mathrm{O}$ Interface, J. Phys. Chem. Lett., 2019, 3, 2031-2036.

39 J. P. Merrick, D. Moran and L. Radom, An Evaluation of Harmonic Vibrational Frequency Scale Factors, J. Phys. Chem. A, 2007, 111, 11683-11700.

40 D. O. Kashinski, G. M. Chase, R. G. Nelson, O. E. Di Nallo, A. N. Scales, D. L. Vanderley and E. F. C. Byrd, Harmonic Vibrational Frequencies: Approximate Global Scaling Factors for TPSS, M06, and M11 Functional Families Using Several Common Basis Sets, J. Phys. Chem. A, 2017, 121, 2265-2273. 
41 I. M. Alecu, J. Zheng, Y. Zhao and D. G. Truhlar, Computational Thermochemistry: Scale Factor Databases and Scale Factors for Vibrational Frequencies Obtained from Electronic Model Chemistries, J. Chem. Theory Comput., 2010, 6, 2872-2887.

42 L. Ruiz Pestana, O. Marsalek, T. E. Markland and T. HeadGordon, The Quest for Accurate Liquid Water Properties from First Principles, J. Phys. Chem. Lett., 2018, 9, 5009-5016.

43 L. Ruiz Pestana, N. Mardirossian, M. Head-Gordon and T. Head-Gordon, Ab Initio Molecular Dynamics Simulations of Liquid Water Using High Quality Meta-GGA Functionals, Chem. Sci., 2017, 8, 3554-3565.

44 J. VandeVondele, M. Krack, F. Mohamed, M. Parrinello, T. Chassaing and J. Hutter, Quickstep: Fast and Accurate Density Functional Calculations Using a Mixed Gaussian and Plane Waves Approach, Comput. Phys. Commun., 2005, 167, 103-128.

45 J. Hutter, M. Iannuzzi, F. Schiffmann and J. VandeVondele, Atomistic Simulations of Condensed Matter Systems, Wiley Interdiscip. Rev.: Comput. Mol. Sci., 2014, 4, 15-25.

46 J. P. Perdew, K. Burke and E. Matthias, Generalized Gradient Approximation Made Simple, Phys. Rev. Lett., 1996, 77, 3865-3868.

47 C. Lee, W. Yang and R. G. Parr, Development of the ColleSalvetti Correlation-Energy Formula into a Functional of the Electron Density, Phys. Rev. B: Condens. Matter Mater. Phys., 1988, 37, 785-789.

48 A. D. Becke, Density-Functional Exchange-Energy Approximation with Correct Asymptotic Behavior, Phys. Rev. A: At., Mol., Opt. Phys., 1988, 38, 3098-3100.

49 Y. Zhang and W. Yang, Comment on "Generalized Gradient Approximation Made Simple.”, Phys. Rev. Lett., 1998, 80, 890.

50 Y. Zhao and D. G. Truhlar, A New Local Density Functional for Main-Group Thermochemistry, Transition Metal Bonding, Thermochemical Kinetics, and Noncovalent Interactions, J. Chem. Phys., 2006, 125, 194101.

51 N. Mardirossian and M. Head-Gordon, Mapping the Genome of Meta-Generalized Gradient Approximation Density Functionals: The Search for B97M-V, J. Chem. Phys., 2015, 142, 074111.

52 A. D. Becke, Density-functional Thermochemistry. III. The Role of Exact Exchange, J. Chem. Phys., 1993, 98, 5648-5652.

53 P. J. Stephens, F. J. Devlin, C. F. Chabalowski and M. J. Frisch, $A b$ Initio Calculation of Vibrational Absorption and Circular Dichroism Spectra Using Density Functional Force Fields, J. Phys. Chem., 1994, 98, 11623-11627.

54 S. H. Vosko, L. Wilk and M. Nusair, Accurate SpinDependent Electron Liquid Correlation Energies for Local Spin Density Calculations: A Critical Analysis, Can. J. Phys., 1980, 58, 1200-1211.

55 C. Adamo and V. Barone, Toward Reliable Density Functional Methods without Adjustable Parameters: The PBE0 Model, J. Chem. Phys., 1999, 110, 6158-6170.

56 J. Heyd, G. E. Scuseria and M. Ernzerhof, Hybrid Functionals Based on a Screened Coulomb Potential, J. Chem. Phys., 2003, 118, 8207-8215.
57 A. V. Krukau, O. A. Vydrov, A. F. Izmaylov and G. E. Scuseria, Influence of the Exchange Screening Parameter on the Performance of Screened Hybrid Functionals, J. Chem. Phys., 2006, 125, 224106.

58 M. Guidon, J. Hutter and J. VandeVondele, Auxiliary Density Matrix Methods for Hartree-Fock Exchange Calculations, J. Chem. Theory Comput., 2010, 6, 2348-2364.

59 S. Grimme, Semiempirical GGA-Type Density Functional Constructed with a Long-Range Dispersion Correction, J. Comput. Chem., 2006, 27, 1787-1799.

60 S. Grimme, J. Antony, S. Ehrlich and H. Krieg, A Consistent and Accurate $\mathrm{Ab}$ Initio Parametrization of Density Functional Dispersion Correction (DFT-D) for the 94 Elements $\mathrm{H}$ $\mathrm{Pu}$, J. Chem. Phys., 2010, 132, 154104.

61 S. Grimme, S. Ehrlich and L. Goerigk, Effect of the Damping Function in Dispersion Corrected Density Functional Theory, J. Comput. Chem., 2011, 32, 1456-1465.

62 D. G. A. Smith, L. A. Burns, K. Patkowski and C. D. Sherrill, Revised Damping Parameters for the D3 Dispersion Correction to Density Functional Theory, J. Phys. Chem. Lett., 2016, 7, 2197-2203.

63 M. Dion, H. Rydberg, E. Schröder, D. C. Langreth and B. I. Lundqvist, van der Waals Density Functional for General Geometries, Phys. Rev. Lett., 2004, 92, 246401.

64 G. Román-Pérez and J. M. Soler, Efficient Implementation of a van der Waals Density Functional: Application to DoubleWall Carbon Nanotubes, Phys. Rev. Lett., 2009, 103, 096102.

65 R. Sabatini, T. Gorni and S. de Gironcoli, Nonlocal van der Waals Density Functional Made Simple and Efficient, Phys. Rev. B: Condens. Matter Mater. Phys., 2013, 87, 041108.

66 O. A. Vydrov and T. Van Voorhis, Nonlocal van der Waals Density Functional: The Simpler the Better, J. Chem. Phys, 2010, 133, 244103.

67 M. F. Kropman, H.-K. Nienhuys, S. Woutersen and H. J. Bakker, Vibrational Relaxation and Hydrogen-Bond Dynamics of HDO: $\mathrm{H}_{2} \mathrm{O}$, J. Phys. Chem. A, 2001, 105, 4622-4626.

68 S. Goedecker, M. Teter and J. Hutter, Separable Dual-Space Gaussian Pseudopotentials, Phys. Rev. B: Condens. Matter Mater. Phys., 1996, 54, 1703-1710.

69 G. Bussi, D. Donadio and M. Parrinello, Canonical Sampling through Velocity Rescaling, J. Chem. Phys., 2007, 126.

70 J. Sun, R. C. Remsing, Y. Zhang, Z. Sun, A. Ruzsinszky, H. Peng, Z. Yang, A. Paul, U. Waghmare and X. Wu, et al., Accurate First-Principles Structures and Energies of Diversely Bonded Systems from an Efficient Density Functional, Nat. Chem., 2016, 8, 831-836.

71 J. Sun, A. Ruzsinszky and J. Perdew, Strongly Constrained and Appropriately Normed Semilocal Density Functional, Phys. Rev. Lett., 2015, 115, 036402.

72 P. Giannozzi, S. Baroni, N. Bonini, M. Calandra, R. Car, C. Cavazzoni, D. Ceresoli, G. L. Chiarotti, M. Cococcioni and I. Dabo, et al., QUANTUM ESPRESSO: A Modular and Open-Source Software Project for Quantum Simulations of Materials, J. Phys.: Condens. Matter, 2009, 21, 395502.

73 P. Giannozzi, O. Andreussi, T. Brumme, O. Bunau, M. Buongiorno Nardelli, M. Calandra, R. Car, C. Cavazzoni, 
D. Ceresoli and M. Cococcioni, et al., Advanced Capabilities for Materials Modelling with Quantum ESPRESSO, J. Phys.: Condens. Matter, 2017, 29, 465901.

74 D. Vanderbilt, Optimally Smooth Norm-Conserving Pseudopotentials, Phys. Rev. B: Condens. Matter Mater. Phys., 1985, 32, 8412-8415.

75 D. R. Hamann, M. Schlüter and C. Chiang, Norm-Conserving Pseudopotentials, Phys. Rev. Lett., 1979, 43, 1494-1497.

76 W. G. Hoover, Canonical Dynamics: Equilibrium Phase-Space Distributions, Phys. Rev. A: At., Mol., Opt. Phys., 1985, 31, 1695-1697.

77 G. J. Martyna, M. L. Klein and M. Tuckerman, Nosé-Hoover Chains: The Canonical Ensemble via Continuous Dynamics, J. Chem. Phys., 1992, 97, 2635-2643.

78 I.-F. W. Kuo, C. J. Mundy, M. J. McGrath and J. I. Siepmann, Time-Dependent Properties of Liquid Water: A Comparison of Car-Parrinello and Born-Oppenheimer Molecular Dynamics Simulations, J. Chem. Theory Comput., 2006, 2, 1274-1281.

79 M. Chen, H.-Y. Ko, R. C. Remsing, M. F. Calegari Andrade, B. Santra, Z. Sun, A. Selloni, R. Car, M. L. Klein and J. P. Perdew, et al., Ab Initio Theory and Modeling of Water, Proc. Natl. Acad. Sci. U. S. A., 2017, 114, 10846-10851.

80 R. Khatib, T. Hasegawa, M. Sulpizi, E. H. G. Backus, M. Bonn and Y. Nagata, Molecular Dynamics Simulations of SFG Librational Modes Spectra of Water at the Water-Air Interface, J. Phys. Chem. C, 2016, 120, 18665-18673.

$81 \mathrm{H}$. Ito and Y. Tanimura, Simulating Two-Dimensional Infrared-Raman and Raman Spectroscopies for Intermolecular and Intramolecular Modes of Liquid Water, J. Chem. Phys., 2016, 144, 074201.

82 T. Hasegawa and Y. Tanimura, A Polarizable Water Model for Intramolecular and Intermolecular Vibrational Spectroscopies, J. Phys. Chem. B, 2011, 115, 5545-5553.

83 Y. Nagata, T. Hasegawa, E. H. G. Backus, K. Usui, S. Yoshimune, T. Ohto and M. Bonn, The Surface Roughness, but Not the Water Molecular Orientation Varies with Temperature at the Water-Air Interface, Phys. Chem. Chem. Phys., 2015, 17, 23559-23564.
84 J. Schaefer, E. H. G. Backus, Y. Nagata and M. Bonn, Both Inter- and Intramolecular Coupling of $\mathrm{O}-\mathrm{H}$ Groups Determine the Vibrational Response of the Water/Air Interface, J. Phys. Chem. Lett., 2016, 7, 4591-4595.

85 S. A. Corcelli and J. L. Skinner, Infrared and Raman Line Shapes of Dilute HOD in Liquid $\mathrm{H}_{2} \mathrm{O}$ and $\mathrm{D}_{2} \mathrm{O}$ from 10 to $90{ }^{\circ} \mathrm{C}, \mathrm{J}$. Phys. Chem. A, 2005, 109, 6154-6165.

86 H. Liu, Y. Wang and J. M. Bowman, Quantum Local Monomer IR Spectrum of Liquid $\mathrm{D}_{2} \mathrm{O}$ at $300 \mathrm{~K}$ from 0 to 4000 $\mathrm{cm}^{-1}$ Is in Near-Quantitative Agreement with Experiment, J. Phys. Chem. B, 2016, 10, 2824-2828.

87 H. Liu, Y. Wang and J. M. Bowman, Quantum Calculations of the IR Spectrum of Liquid Water Using $\mathrm{Ab}$ Initio and Model Potential and Dipole Moment Surfaces and Comparison with Experiment, J. Chem. Phys., 2015, 142, 194502.

88 F. Tang, T. Ohto, S. Sun, J. R. Rouxel, S. Imoto, E. H. G. Backus, S. Mukamel, M. Bonn and Y. Nagata, Molecular Structure and Modeling of Water-Air and IceAir Interfaces Monitored by Sum-Frequency Generation, Chem. Rev., 2020, 120, 3633-3667.

$89 \mathrm{X} . \mathrm{Xu}, \mathrm{Y} . \mathrm{R}$. Shen and C. Tian, Phase-Sensitive Sum Frequency Vibrational Spectroscopic Study of Air/Water Interfaces: $\mathrm{H}_{2} \mathrm{O}, \mathrm{D}_{2} \mathrm{O}$, and Diluted Isotopic Mixtures, J. Chem. Phys., 2019, 150, 144701.

90 S. Y. Venyaminov and F. G. Prendergast, Water $\left(\mathrm{H}_{2} \mathrm{O}\right.$ and $\left.\mathrm{D}_{2} \mathrm{O}\right)$ Molar Absorptivity in the $1000-4000 \mathrm{~cm}^{-1}$ Range and Quantitative Infrared Spectroscopy of Aqueous Solutions, Anal. Biochem., 1997, 248, 234-245.

91 I. R. Craig and D. E. Manolopoulos, Quantum Statistics and Classical Mechanics: Real Time Correlation Functions from Ring Polymer Molecular Dynamics, J. Chem. Phys., 2004, 121, 3368-3373.

92 J. Cao and G. A. Voth, The Formulation of Quantum Statistical Mechanics Based on the Feynman Path Centroid Density. II. Dynamical Properties, J. Chem. Phys., 1994, 100, 5106-5117.

93 A. Luzar and D. Chandler, Hydrogen-Bond Kinetics in Liquid Water, Nature, 1996, 379, 55-57. 